Provided for non-commercial research and education use. Not for reproduction, distribution or commercial use.

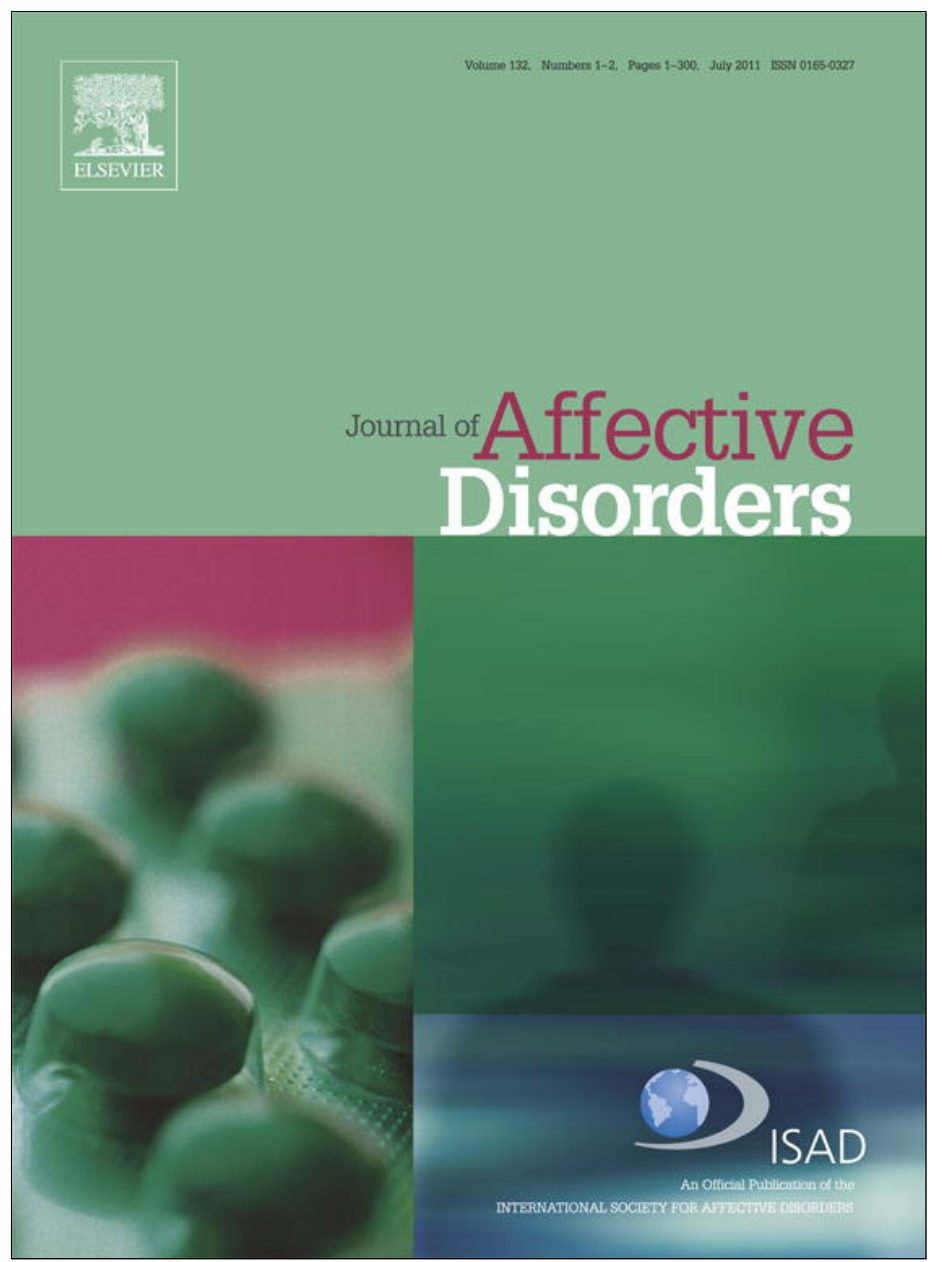

This article appeared in a journal published by Elsevier. The attached copy is furnished to the author for internal non-commercial research and education use, including for instruction at the authors institution and sharing with colleagues.

Other uses, including reproduction and distribution, or selling or licensing copies, or posting to personal, institutional or third party websites are prohibited.

In most cases authors are permitted to post their version of the article (e.g. in Word or Tex form) to their personal website or institutional repository. Authors requiring further information regarding Elsevier's archiving and manuscript policies are encouraged to visit:

http://www.elsevier.com/copyright 
Research report

\title{
Anxiety and depression symptoms in women and men from early pregnancy to 3-months postpartum: Parity differences and effects
}

\author{
Bárbara Figueiredo*, Ana Conde \\ School of Psychology, University of Minho, Portugal
}

\section{A R T I C L E I N F O}

\section{Article history:}

Received 7 September 2010

Received in revised form 9 February 2011

Accepted 9 February 2011

Available online 21 March 2011

\section{Keywords:}

Anxiety

Depression

Gender

Parity

Pregnancy

Postpartum

Women/men

First and second-time time parents

\begin{abstract}
A B S T R A C T
This study aimed to investigate both anxiety and depression symptoms from early pregnancy to 3-months postpartum, comparing women and men and first and second-time parents. Methods: A sample of 260 Portuguese couples $(N=520)$, first or second-time parents, recruited in an Obstetrics Out-patients Unit, filled in the State-Anxiety Inventory (STAI-S) and the Edinburgh Post-Natal Depression Scale (EPDS) at the 1st, 2nd and 3rd pregnancy trimesters, childbirth, and 3-months postpartum.

Results: A decrease in anxiety and depression symptoms from early pregnancy to 3-months postpartum was found in both women and men, as well as in first and second-time parents. Men presented less anxiety and depression symptoms than women, but the same pattern of symptoms over time. Second-time parents showed more anxiety and depression symptoms than first-time parents and a different pattern of symptoms over time: an increase in anxiety and depression symptoms from the 3rd trimester to childbirth was observed in first-time parents versus a decrease in second-time parents.

Limitations: The voluntary nature of the participation may have lead to a selection bias; women and men who agreed to participate could be those who presented fewer anxiety and depression symptoms. Moreover, the use of self-report symptom measures does not give us the level of possible disorder in participants.

Conclusions: Anxiety and depression symptoms diminish from pregnancy to the postpartum period in all parents. Patterns of anxiety and depression symptoms from early pregnancy to 3months postpartum are similar in women and men, but somewhat different in first and secondtime parents. Second-time parents should also be considered while studying and intervening during pregnancy and the postpartum.
\end{abstract}

(C) 2011 Elsevier B.V. All rights reserved.

\section{Introduction}

From early pregnancy to the postpartum period, several changes occur simultaneously at biological, psychological and social levels. Adjustment to these changes may place both women and men at-risk for psychological difficulties. Literature has been picturing a particularly difficult transition for firsttime parents. But for second-time parents adjustment and

\footnotetext{
* Corresponding author at: School of Psychology, University of Minho, Campus de Gualtar, 4710-057 Braga, Portugal. Tel.: +351 253 604241; fax: + 351253678987

E-mail address: bbfi@psi.uminho.pt (B. Figueiredo).
}

resultant strain may come from the process of incorporating a new member into a preexisting system and having to take care of two children at the same time.

Empirical studies report high levels of anxiety symptoms in women (Andersson et al., 2006; Breitkopf et al., 2006; DiPietro et al., 2006; Heron et al., 2004; Huizink et al., 2004; Lee et al., 2007) and their partners (Boyce et al., 2007; Condon et al., 2004; Dulude et al., 2002; Perren et al., 2005; Skari et al., 2002) during pregnancy. In both women and men, anxiety levels seem to be higher in the 1 st and 3rd compared to the 2nd pregnancy trimesters (Lee et al., 2007; Teixeira et al., 2009), and higher during pregnancy/childbirth compared to the postpartum period (Andersson et al., 2006; Breitkopf et al., 
2006; Condon et al., 2004; DiPietro et al., 2006; Heron et al., 2004; Skari et al., 2002).

High levels of depression symptoms have been reported as well, during pregnancy in women (Andersson et al., 2006; Breitkopf et al., 2006; Bunevicius et al., 2009; Caliskan et al., 2007; DiPietro et al., 2008; Evans et al., 2001; Felice et al., 2004; Figueiredo et al., 2007; Heron et al., 2009; Lau and Chan, 2009; Limlomwongse and Liabsuetrakul, 2006; Ross and McLean, 2006; Skouteris et al., 2009; Westdahl et al., 2007; Wu et al., 2002) and their partners (Areias et al., 1996; Buist et al., 2003; Condon et al., 2004; Deater-Deckard et al., 1998; Dulude et al., 2002; Morse et al., 2000; Perren et al., 2005), plus in both postpartum mothers (Dietz et al., 2007; Dørheim et al., 2009; Gorman et al., 2004; Xie et al., 2009) and fathers (Ballard and Davis, 1996; Edhborg et al., 2005). Following many years of interest in postpartum depression, research has been more recently picturing a decrease in depression from the 1st to the 3rd pregnancy trimester (Andersson et al., 2006; Breitkopf et al., 2006; Bunevicius et al., 2009; Felice et al., 2004; DiPietro et al., 2008; Ross and McLean, 2006; Teixeira et al., 2009), and from pregnancy to the postpartum period (Evans et al., 2001; Felice et al., 2004; Figueiredo et al., 2007; Heron et al., 2009; Kinsella and Monk, 2009; Lau and Chan, 2009; Limlomwongse and Liabsuetrakul, 2006; Skouteris et al., 2009), in women, as well as in men (Buist et al., 2003; Condon et al., 2004; Dulude et al., 2002; Morse et al., 2000; Perren et al., 2005). However, higher rates of depression after childbirth compared to pregnancy are still being found in women (Dietz et al., 2007; Gorman et al., 2004), and in men, particularly at the end of the first postpartum year (Matthey et al., 2000).

Usually, women have been described as presenting higher anxiety than their partner, either before (Dulude et al., 2002) or after childbirth (Ballard and Davis, 1996; Skari et al., 2002), but some studies do not show gender differences in levels of postpartum anxiety (Dulude et al., 2002). During pregnancy women have been reported with higher depression levels than men (Dulude et al., 2002; Matthey et al., 2000; Morse et al., 2000; Perren et al., 2005), which is also controversial with studies showing no significant gender differences (Field et al., 2006). Reports of two to three times more postpartum depressed mothers than fathers (Areias et al., 1996; Ballard and Davis, 1996; Edhborg et al., 2005; Matthey et al., 2000; Morse et al., 2000; Perren et al., 2005) are also controversial with research showing no gender differences in postpartum depression rates (Dulude et al., 2002).

First-time mothers and fathers have been described as presenting higher anxiety than second-time mothers and fathers, during pregnancy and the postpartum (Hung, 2004; Giakoumaki et al., 2009), with some studies showing the reverse in pregnancy (DiPietro et al., 2008) and at postpartum (Skari et al., 2002). No differences were found in literature in women's anxiety according to parity in pregnancy (Lee et al., 2007) and postpartum (Breitkopf et al., 2006; DiPietro et al., 2006) as well.

No differences in depression levels according to parity have been generally reported in women during pregnancy (Breitkopf et al., 2006; Bunevicius et al., 2009; Dietz et al., 2007; Lee et al., 2007; Westdahl et al., 2007; Wu et al., 2002) and the postpartum period (Dietz et al., 2007; Wenzel et al., 2005). However, some studies report either lower depression levels in first-time compared to second-time mothers during pregnancy (DiPietro et al., 2008; Vliegen et al., 2006) or the postpartum (Ho-Yen et al., 2007; Righetti-Veltema et al., 1998; Skari et al., 2002), and higher in first-time compared to second-time mothers during the postpartum (Dørheim et al., 2009). Studies have been mostly targeting first-time fathers (e.g., Areias et al., 1996; Matthey et al., 2000; Morse et al., 2000; Perren et al., 2005), and so not much data is available about the association between parity and depression in men, with the exception of one study showing high levels of depression during pregnancy in second-time compared to first-time fathers (Condon and Esuvaranathan, 1990).

High levels of anxiety and depression symptoms have been reported during pregnancy and the postpartum period, typically depression in women 3-months after childbirth. However, a decrease from pregnancy to the postpartum period in anxiety and depression symptoms has been pointed out more recently, in women (Andersson et al., 2006; Breitkopf et al., 2006; DiPietro et al., 2008; Evans et al., 2001; Felice et al., 2004; Field et al., 2008; Figueiredo et al., 2007; Heron et al., 2004, 2009; Limlomwongse and Liabsuetrakul, 2006; Ross et al., 2003; Skouteris et al., 2009) as in men (Buist et al., 2003; Condon et al., 2004; Dulude et al., 2002; Perren et al., 2005; Skari et al., 2002). Usually studies show more anxiety and depression symptoms in women than in their partners either before or after childbirth (Ballard and Davis, 1996; Condon and Esuvaranathan, 1990; Edhborg et al., 2005; Matthey et al., 2000; Morse et al., 2000; Perren et al., 2005; Skari et al., 2002). Controversial results in the literature regarding anxiety and depression during pregnancy and the postpartum period are mainly regarding first and secondtime parents. Studies usually found no differences in anxiety and depression symptoms between primipara and multipara mothers (Breitkopf et al., 2006; Bunevicius et al., 2009; Dietz et al., 2007; Figueiredo et al., 2007; Lee et al., 2007; Wenzel et al., 2005; Westdahl et al., 2007; Wu et al., 2002); though, more (Dørheim et al., 2009; Gameiro et al., 2009; Giakoumaki et al., 2009; Hung, 2004), as well as less symptoms (DiPietro et al., 2008; Ho-Yen et al., 2007; Righetti-Veltema et al., 1998; Skari et al., 2002; Vliegen et al., 2006) in first-time mothers than in second-time mothers have also been reported.

Patterns of anxiety and depression symptoms change throughout pregnancy and the postpartum period according to gender and parity have not been as highly studied, and therefore are the principal novelties of the present study. Additionally, studies targeting men's psychological adjustment during pregnancy and the postpartum period have almost only considered first-time fathers (Areias et al., 1996; Boyce et al., 2007; Buist et al., 2003; Condon et al., 2004; Matthey et al., 2000; Morse et al., 2000; Perren et al., 2005). Some controversial results in the literature may come from the fact that research has been mostly designed to study postpartum depression in first-time mothers, while men and second-time parents may have a different pattern of symptoms over time. Studying simultaneously women and men and first and second-time parents from early pregnancy to 3-months postpartum could help to explain controversial results concerning levels of anxiety and depression symptoms throughout pregnancy and the postpartum period.

This study was aimed to investigate anxiety and depression symptoms in first and second-time mothers and fathers 
at the 1st, 2nd and 3rd pregnancy trimesters, childbirth and 3-months postpartum, chiefly looking at parity differences and effects.

We hypothesized that the transition to parenthood is mainly the same developmental process, generating the same psychological challenges and difficulties for women and men and for first and second-time parents. But we also hypothesized that some different patterns of psychological adjustment throughout pregnancy and the postpartum period may occur in women and in men and in first and second-time parents, according to specific strains and difficulties that the transition may have implied for women and men and for first and second-time parents (e.g., DiPietro et al., 2008; Gameiro et al., 2009; Teixeira et al., 2009).

\section{Methods}

\subsection{Participants}

Three hundred pregnant women were randomly recruited in an Obstetrics Out-patients Unit (Porto, Portugal) (84\% of the contacted pregnant women). The exclusion criteria were: not reading or writing Portuguese and multiple gestations. A sample of 260 couples $(N=520)$ was considered, when the partner also agreed to participate; $91.4 \%$ of women and $82.6 \%$ of men completed all five assessments.

Nearly all the participants were Portuguese (91.4\%). The majority of participants was aged between 20 and 39 years old (85.9\%); belonged to low or medium-low socio-economic level (57.4\%); and were employed in manual (qualified or not qualified) professions (57.4\%). Participants were married (55.8\%) or cohabiting (32.6\%), only $10.7 \%$ were single and $0.6 \%$ divorced. More than half of the women $(54.1 \%)$ and men (56.1\%) were first-time parents, but $44.9 \%$ were second-time parents.
Women were younger $[\chi 2(3)=22.455, p=.000]$, more unemployed $[\chi 2(2)=22.597, p=.000]$ and more educated than men $[\chi 2(2)=11.574, p=.003]$. First-time parents were younger $[\chi 2(3)=50.753, p=.000]$, more educated $[\chi 2(2)$ $8.096, p=.017]$, and more of them were single [ $\chi 2(2) 24.653$, $p=.017]$ than second-time parents. No significant differences were obtained for the remaining socio-demographics (see Table 1).

At the first contact, $11.7 \%$ of the participants had STAI-S scores equal or higher than 45 (STAI-S $\geq 45$ ) (women $=$ $13.1 \%$; men $=10.3 \%$; first-time parents $=10.3 \%$; second-time parents $=13.4 \%$ ) and $15.9 \%$ of the participants had EPDS scores equal or higher than 10 (EPDS $\geq 10$ ) (women $=20 \%$; men $=11.5 \%$; first-time parents $=13.2 \%$; second-time parents $=19.2 \%)$. Participants with STAI-S $\geq 45$ were more likely to be unemployed $[\chi 2(2)=10.739, p=.005]$. Participants with EPDS $\geq 10$ were more likely to be younger [ $\chi 2(3) 14.876, p=.002]$, unemployed $[\chi 2(2)=30.733$, $p=.000]$ and less educated $[\chi 2(2)=6.750, p=.034]$. No significant association was obtained for the reaming sociodemographics.

\subsection{Measures}

\subsubsection{Socio-demographic questionnaire}

Information about the participants (e.g., age, occupational status, and marital status) was collected using a sociodemographic questionnaire.

2.2.2. State-anxiety inventory (STAI-S, Spielberger et al., 1983)

A twenty-item self-report scale was used for measuring the temporary condition of state-anxiety (anxiety in a specific situation). STAI-S Portuguese version has shown good internal consistence (Cronbach Alpha $=0.87-0.93$ ) (Biaggio et al., 1976).

Table 1

Socio-demographics (women and men, 1st and 2nd time parents, and total sample).

\begin{tabular}{|c|c|c|c|c|c|c|}
\hline & & \multirow{2}{*}{$\frac{\text { Women }}{n=260}$} & \multirow{2}{*}{$\frac{\text { Men }}{n=260}$} & \multirow{2}{*}{$\frac{1 \text { st time }}{n=320}$} & \multirow{2}{*}{$\frac{\text { 2nd time }}{n=200}$} & \multirow{2}{*}{$\frac{\text { Total }}{N=520}$} \\
\hline & & & & & & \\
\hline & & $\%$ & $\%$ & $\%$ & $\%$ & $\%$ \\
\hline \multirow[t]{4}{*}{ Age (years) } & $\leq 19$ & 11.5 & 4.2 & 12.4 & 2.6 & 7.9 \\
\hline & $20-29$ & 47.0 & 38.9 & 51.6 & 33.3 & 42.9 \\
\hline & $30-39$ & 39.2 & 46.9 & 33.9 & 55.4 & 43.1 \\
\hline & $\geq 40$ & 2.3 & 10.0 & 2.1 & 8.7 & 6.1 \\
\hline \multirow[t]{4}{*}{ Matrimonial status } & Cohabiting & 32.6 & 32.5 & 35.8 & 28.5 & 32.5 \\
\hline & Married & 55.8 & 55.7 & 49.7 & 63.2 & 55.8 \\
\hline & Single & 11.2 & 10.2 & 14.5 & 6.1 & 10.7 \\
\hline & Divorced & 0.4 & 1.6 & 0 & 2.2 & 1.0 \\
\hline \multirow[t]{5}{*}{ Socio-economic level } & High & 16.4 & 9.3 & 15.6 & 8.9 & 12.5 \\
\hline & Medium high & 7.4 & 14.0 & 9.4 & 13.0 & 11.1 \\
\hline & Medium & 19.6 & 18.6 & 16.5 & 21.9 & 19.0 \\
\hline & Medium low & 26.4 & 30.3 & 30.4 & 26.5 & 28.6 \\
\hline & Low & 30.2 & 27.8 & 28.1 & 27.7 & 28.8 \\
\hline \multirow{2}{*}{ Occupational status } & Unemployed & 21.6 & 9.0 & 20.0 & 13.4 & 15.4 \\
\hline & Household/student & 6.6 & 2.0 & 5.3 & 3.1 & 4.2 \\
\hline \multirow[t]{4}{*}{ Profession } & Non manual qualified & 23.8 & 23.3 & 25.0 & 21.9 & 23.6 \\
\hline & Non manual not qualified & 19.6 & 18.5 & 16.5 & 21.9 & 19.0 \\
\hline & Manual qualified & 26.4 & 30.4 & 30.4 & 26.5 & 28.6 \\
\hline & Manual not qualified & 30.2 & 27.8 & 28.1 & 29.7 & 28.8 \\
\hline \multirow[t]{3}{*}{ Schooling (years) } & $<9$ & 26.9 & 38.6 & 29.3 & 37.8 & 32.8 \\
\hline & $9-12$ & 54.3 & 47.9 & 53.0 & 50.0 & 51.0 \\
\hline & $>12$ & 18.8 & 13.5 & 17.7 & 12.2 & 16.2 \\
\hline
\end{tabular}


2.2.3. Edinburgh Postnatal Depression Scale (EPDS, Cox et al., 1987)

A self-report questionnaire composed of 10-items addressing depression symptoms within the previous seven days. EPDS Portuguese version has shown good internal consistency (Cronbach Alpha = 0.85) (Figueiredo et al., 2007).

\subsection{Procedures}

This research received previous approval from the institution review board and was conducted in accordance with the Helsinki Declaration. Participants were recruited in an Obstetrics Out-patients Unit at their first appointment. The aims and the procedures of the study were explained. After signing an informed consent form, the socio-demographic questionnaire was separately filled-out by the pregnant woman and partner, and the STAI-S and EPDS was given to them (between weeks 8 and 14). The STAI-S and EPDS were once again filled-out during the 2nd (between weeks 20 and 24) and 3rd pregnancy trimester (between weeks 30 and 34), as well as at childbirth (between days 1 and 3) and at 3-months postpartum (between weeks 10 and 14).

\subsection{Statistical analysis}

Repeated measures analysis of variance (ANOVA) was applied in order to study changes in anxiety and depression symptoms from early pregnancy to 3-months postpartum. Gender and parity were included as potential modifiers (between-subjects factors), in order to compare women and men and first and second-time parents. STAI-S and EPDS scores were included as measures of the same within-subject factor with five levels (1st, 2nd and 3rd pregnancy trimesters, childbirth and 3-months postpartum). As gender and/or parity significant differences were found concerning age, occupational status, schooling, and matrimonial status, these variables were included as covariates in order to control their effects. Main effects and interaction terms were tested. Post hoc contrasts were used to determine the nature of trends over time. Pairwise comparisons with Bonferroni correction were applied to analyze mean differences in anxiety and depression symptoms comparing all measuring time points. In order to explore the same aim respectively in women, men, and first and secondtime parents, independent repeated measures ANOVAs were implemented. When women and men were analyzed, the effect of parity was controlled; when first and second-time parents were analyzed, the effect of gender was controlled. Independent samples $t$-test analysis were implemented to explore gender and parity differences in anxiety and depression symptoms at each of the measuring time points and paired samples $t$-test were used to analyze differences in anxiety and depression symptoms between measuring time points.

\section{Results}

\subsection{From early pregnancy compared to 3-months postpartum}

Repeated measures ANOVA revealed significant changes over pregnancy and the postpartum period $[\mathrm{F}(8,2750) 5.24$, $p<0.001]$, both for anxiety $[\mathrm{F}(4,1376)=6.00, p<0.001]$ and depression symptoms $[\mathrm{F}(4,1376)=7.51, p<0.001]$.

Table 2

Anxiety symptoms from early pregnancy to 3-months postpartum: women and men, 1st and 2nd time parents, and total sample.

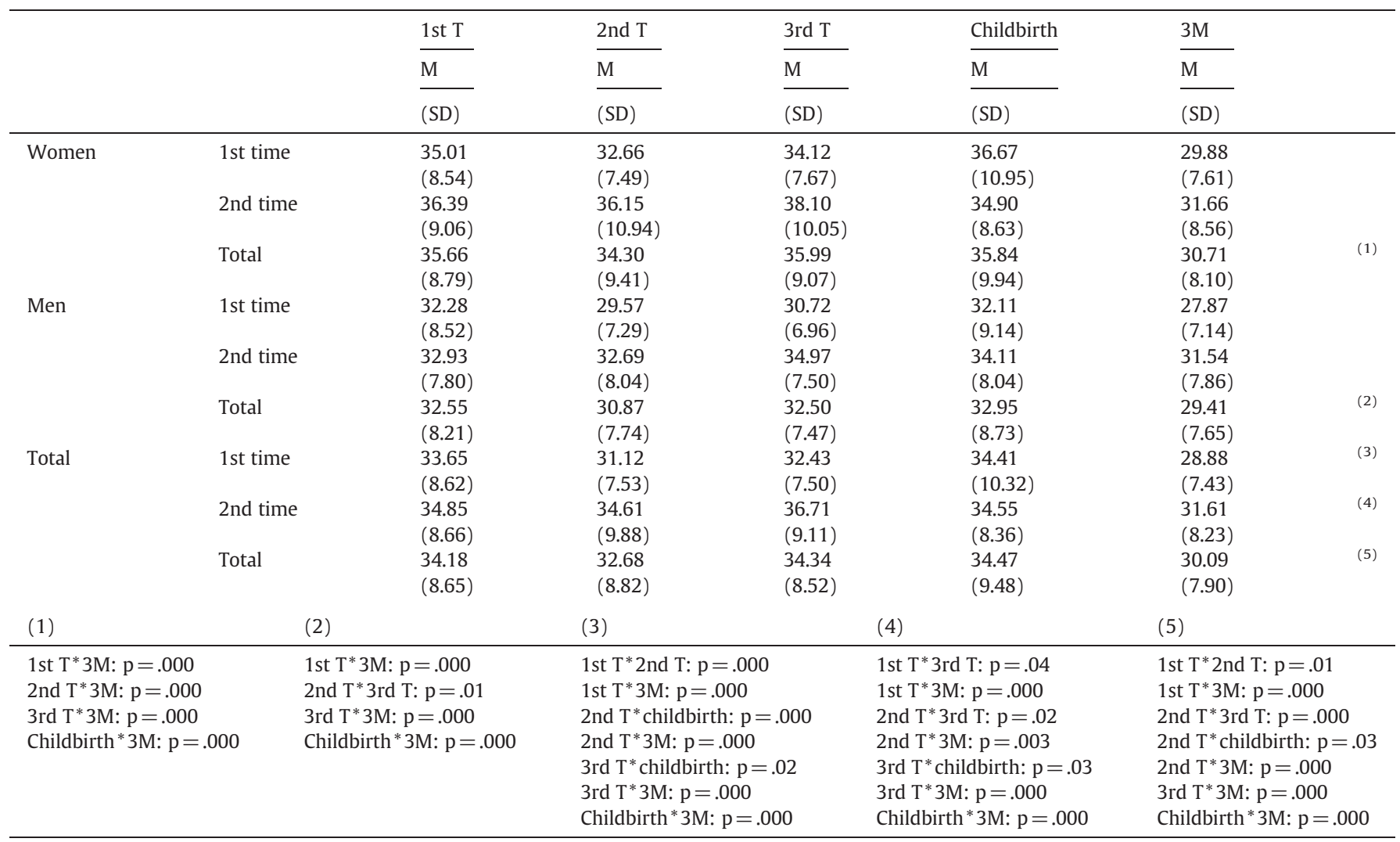


Post hoc contrasts for anxiety symptoms showed a significant increase from the 3rd trimester to childbirth. However, changes observed from the 1st to the 2nd trimester, the 2nd to the 3rd trimester, and from childbirth to 3-months postpartum were not significant. Pair wise comparisons concerning anxiety symptoms revealed significant mean differences between the 1st trimester and: the 2nd trimester and 3-months postpartum; the 2nd trimester and: the 3rd trimester, childbirth and 3-months

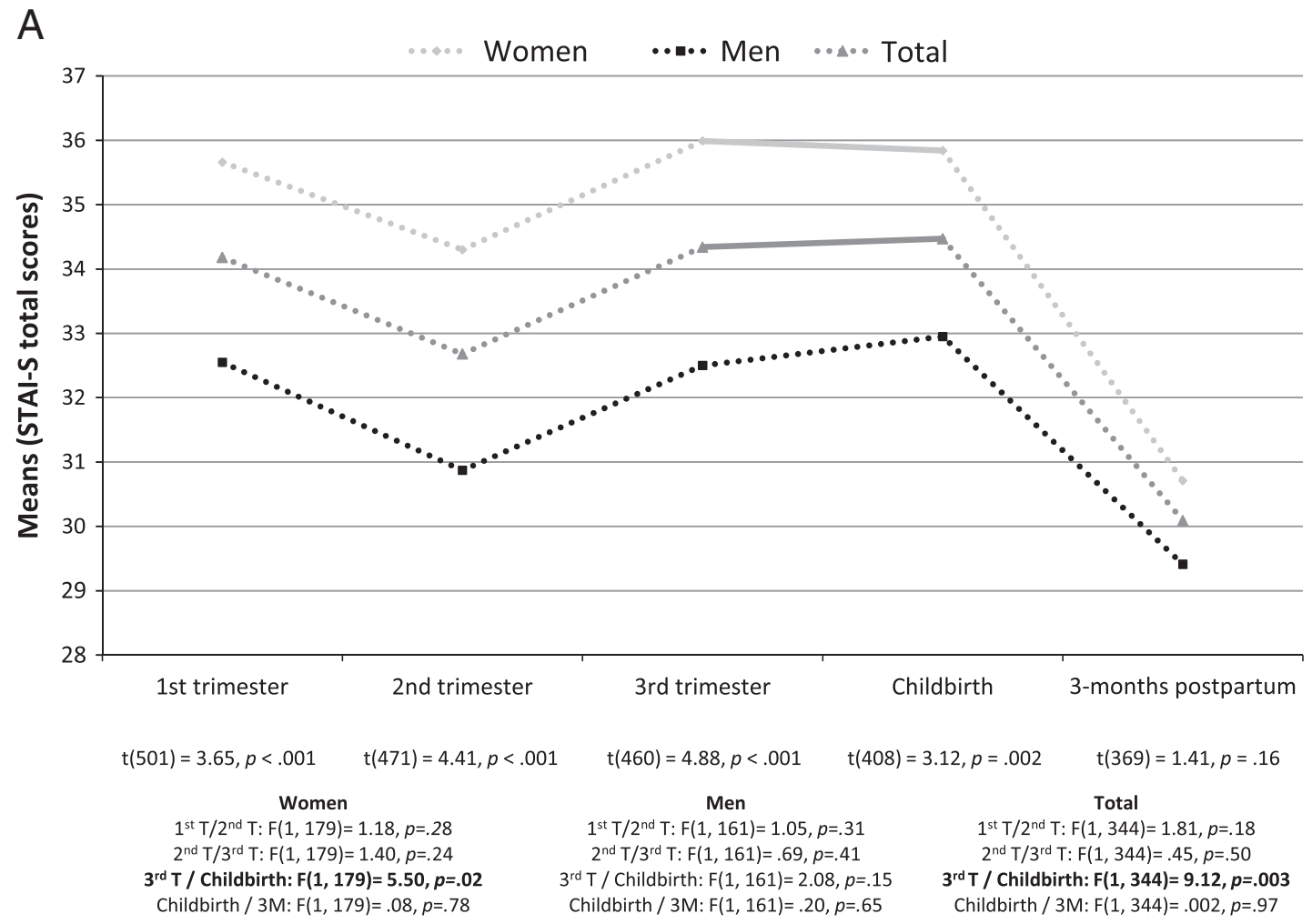

B

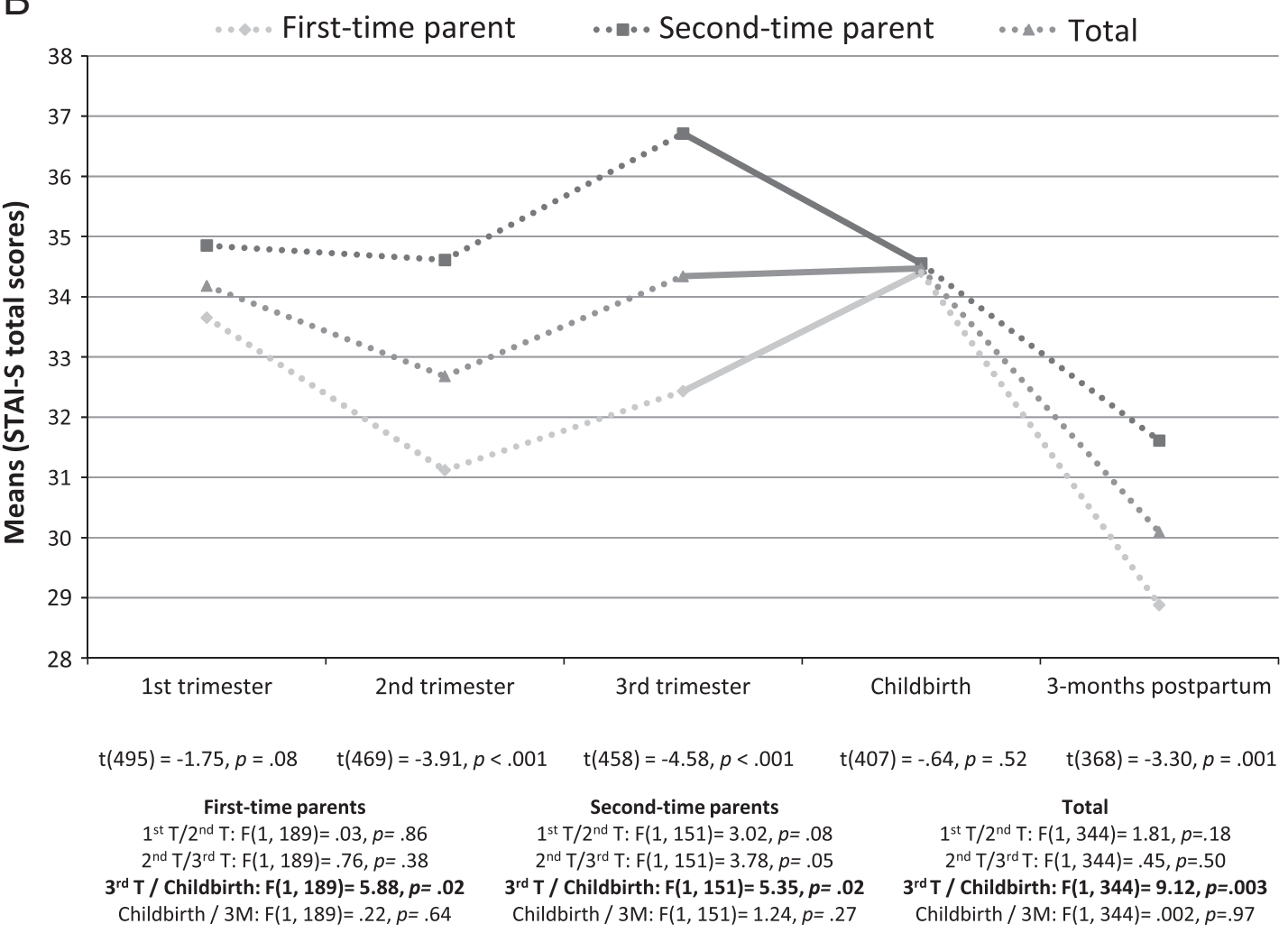

Fig. 1. (A) Anxiety symptoms from early pregnancy to 3-months postpartum: women, men, and total sample. (B) Anxiety symptoms from early pregnancy to 3months postpartum: 1 st and 2 nd time parents, and total sample. 


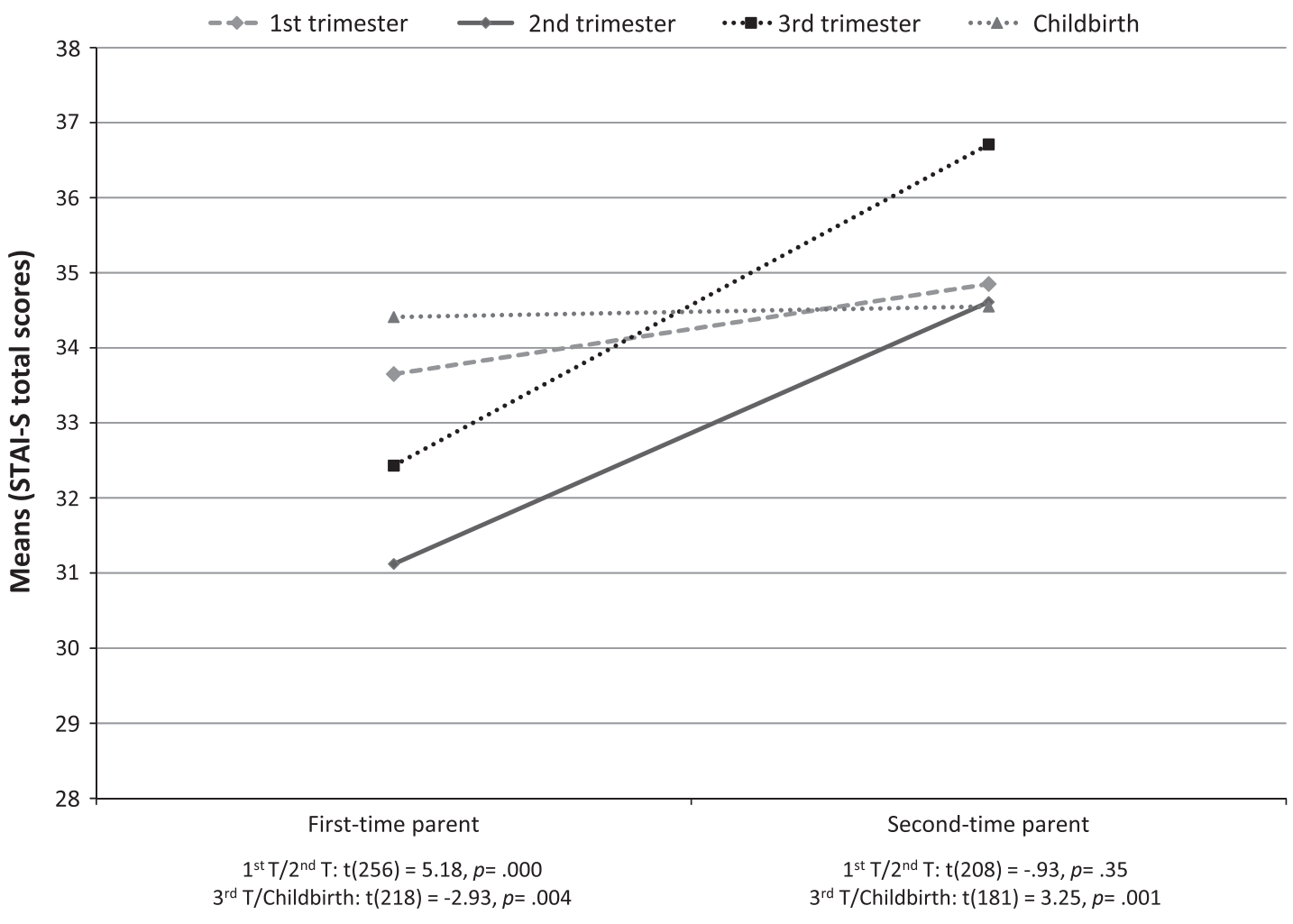

Fig. 2. Interaction between measuring time points and parity for anxiety symptoms.

postpartum; the 3rd trimester and 3-months postpartum; and childbirth and 3-months postpartum (see Table 2/Fig. 1).

Post hoc contrasts for depression symptoms showed a significant decrease from the 1 st to the 2nd trimester. Nevertheless, from: the 2nd to the 3rd trimester; the 3rd trimester to childbirth; and from childbirth to 3-months postpartum, changes were not significant. Pair wise comparisons concerning depression symptoms revealed significant mean differences between the 1st and: the 2nd and 3rd trimesters, childbirth, and 3-months postpartum; and between 3-months postpartum and: the 2 nd and 3rd trimesters, and childbirth (see Fig. 3).

\subsection{Women compared to men}

Significant gender effects $[\mathrm{F}(2,343)=7.78, p<0.001]$ were found in anxiety $[\mathrm{F}(1,344)=13.91, \mathrm{p}<0.001]$ and depression $[F(1,344)=13.54, p<0.001]$ symptoms. Women showed higher anxiety symptoms than men at the 1st, 2nd, and 3rd trimesters, and at childbirth, but not at 3-months postpartum (see Table 2/Fig. 1). Women exhibited more depression symptoms than men in all the considered measuring times points (see Fig. 3). No significant interaction between measuring time points and gender was found $[\mathrm{F}(8$, $2750)=1.73, p=.09]$, for anxiety $[F(3.6,1235.5)=1.74$, $p=.14]$, nor for depression symptoms $[\mathrm{F}(3.6,1238.8) 1.45$, $p=.22]$.

Post hoc contrasts for women's anxiety symptoms showed significant changes from the 3rd trimester to childbirth, but not from the 1 st to the 2nd trimester, the 2nd to the 3rd trimester, and from childbirth to 3-months postpartum. Pair wise comparisons applied to women's anxiety symptoms revealed significant mean differences between 3-months postpartum and: the 1st, the 2nd and the 3rd trimesters, and childbirth (see Table 2/Fig. 1). Significant changes were also found in women's depression symptoms from the 1 st to the 2nd trimester, but not from the 2nd to the 3rd trimester, the 3rd trimester to childbirth, and from childbirth to 3months postpartum. Pair wise comparisons applied to women's depression symptoms revealed significant mean differences between the 1st trimester and: the 3rd trimester and 3-months postpartum; and between 3-months postpartum and: the 2nd trimester and childbirth (see Fig. 3).

In men, no significant changes in anxiety symptoms between any of the considered measuring time points were obtained. Pair wise comparisons applied to men's anxiety symptoms showed significant mean differences between 3months postpartum and: the 1st and 3rd trimesters, and childbirth; and between the 2nd and the 3rd trimester (see Table 2/Fig. 1). Significant changes were found in men's depression symptoms from the 1 st to the 2 nd trimester, but not from the 2nd to the 3rd trimester, the 3rd trimester to childbirth, or from childbirth to 3-months postpartum. Pair wise comparisons applied to men's depression symptoms revealed significant mean differences between the $1 \mathrm{st}$ trimester and: the 2nd and the 3rd trimesters, childbirth, and 3-months postpartum (see Fig. 3).

\subsection{First-time compared to second-time parents}

Significant parity effects $[F(2,343)=4.09, p=.02]$ were found in anxiety $[\mathrm{F}(1,344)=7.77, p=.006]$, as well as in depression symptoms $[F(1,344)=6.47, p=.01]$. Secondtime parents showed more anxiety symptoms than first-time 

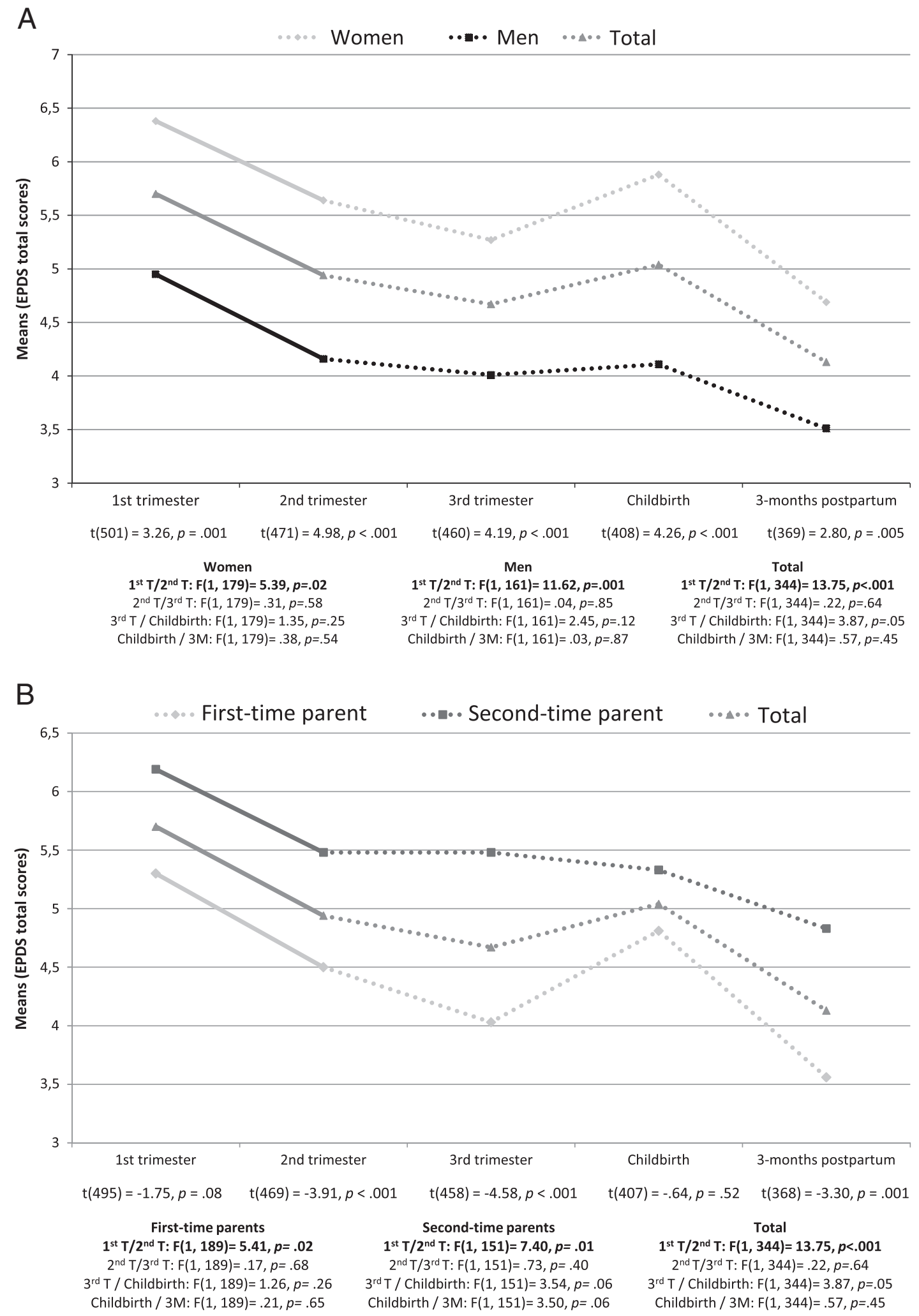

Fig. 3. (A) Depression from early pregnancy to 3-months postpartum: women, men, and total sample. (B) Depression from early pregnancy to 3-months postpartum: 1st and 2nd time parents, and total sample.

parents at the 2nd and 3rd trimesters, and at 3-months postpartum, but not at the 1 st trimester or at childbirth (see Table 2/Fig. 1). Second-time parents showed more depression symptoms than first-time parents at the 1st, 2nd and 3rd trimesters, as well as at 3-months postpartum, but no significant differences were found at childbirth (see Fig. 3).
A significant interaction was found between measuring time points and parity $[\mathrm{F}(8,2750)=4.05, p<0.001]$, both for anxiety $[F(3.6,1235.5)=6.84, p<0.001]$, as well as for depression symptoms $[\mathrm{F}(3.6,1238.8)=3.08, p=.02]$. First-time mothers and fathers showed higher anxiety symptoms at the 1st when compared to the 2 nd trimester, while no significant differences 
were found in second-time parents. Additionally, while firsttime mothers and fathers showed higher anxiety symptoms at childbirth than at the 3rd trimester, second-time parents showed higher anxiety symptoms at the 3rd trimester than at childbirth (see Fig. 2).

For depression symptoms, while first-time parents showed higher depression symptoms at the 2nd than at the 3rd trimester, no significant differences were observed between the 2nd and the 3rd trimesters in second-time mothers and fathers. Additionally, while first-time parents showed higher depression symptoms at childbirth than at the 3rd trimester, second-time parents showed higher depression symptoms at the 3rd trimester comparing to childbirth (see Fig. 4).

No significant interaction was found neither between parity and gender $[\mathrm{F}(2,343)=.23, p=.79]$ nor between measuring time points, parity and gender $[\mathrm{F}(8,2750)=1.17$, $p=.31]$.

Focusing on first-time parents, significant changes in anxiety symptoms were observed from the 3rd trimester to childbirth, but not from the 1 st to the 2 nd trimester, the 2 nd to the 3rd trimester, and from childbirth to 3-months postpartum. Pair wise comparisons applied to first-time parents' anxiety symptoms revealed significant mean differences between the 1 st trimester and: the 2nd trimester and 3-months postpartum; the 2nd trimester and: childbirth and 3-months postpartum; the 3rd trimester and: childbirth and the 3-months postpartum; and between childbirth and 3-months postpartum (see Table 2/Fig. 1). Significant changes were found in first-time parents' depression symptoms from the 1 st to the 2nd trimester, but not from the 2nd to the 3rd trimester, the 3rd trimester to childbirth or from childbirth to 3-months postpartum. Pair wise comparisons applied to first-time parents' depression symptoms revealed significant mean differences between the 1st trimester and: the 2nd and 3rd trimesters, and 3-months postpartum; the 2nd trimester and 3-months postpartum; the 3rd trimester and childbirth; and between childbirth and 3-months postpartum (see Table 3/Fig. 3).

Focusing on second-time parents significant changes were obtained in anxiety symptoms from the 3rd trimester to childbirth, marginally significant differences from the 1 st to the 2nd trimester and from the 2nd to the 3rd trimester, and no significant differences from childbirth to 3-months postpartum. Pair wise comparisons applied to second-time parents' anxiety symptoms revealed significant mean differences between the 1st trimester and: the 3rd trimester and 3-months postpartum; the 2nd trimester and: the 3rd trimester and 3-months postpartum; the 3rd trimester and: childbirth and 3-months postpartum; and between childbirth and 3-months postpartum (see Table 2/Fig. 1). Significant changes were found in secondtime parents' depression symptoms from the 1st to the 2nd trimester, marginally significant differences from the 3rd trimester to childbirth and from childbirth to 3-months postpartum, and no significant differences from the 2nd to the 3rd trimester. Pair wise comparisons applied to secondtime parents' depression symptoms revealed significant mean differences between the 1st trimester and 3-months postpartum (see Table 3/Fig. 3).

\section{Discussion}

Anxiety and depression symptoms were present during the entire studied period, supporting psychological morbidity

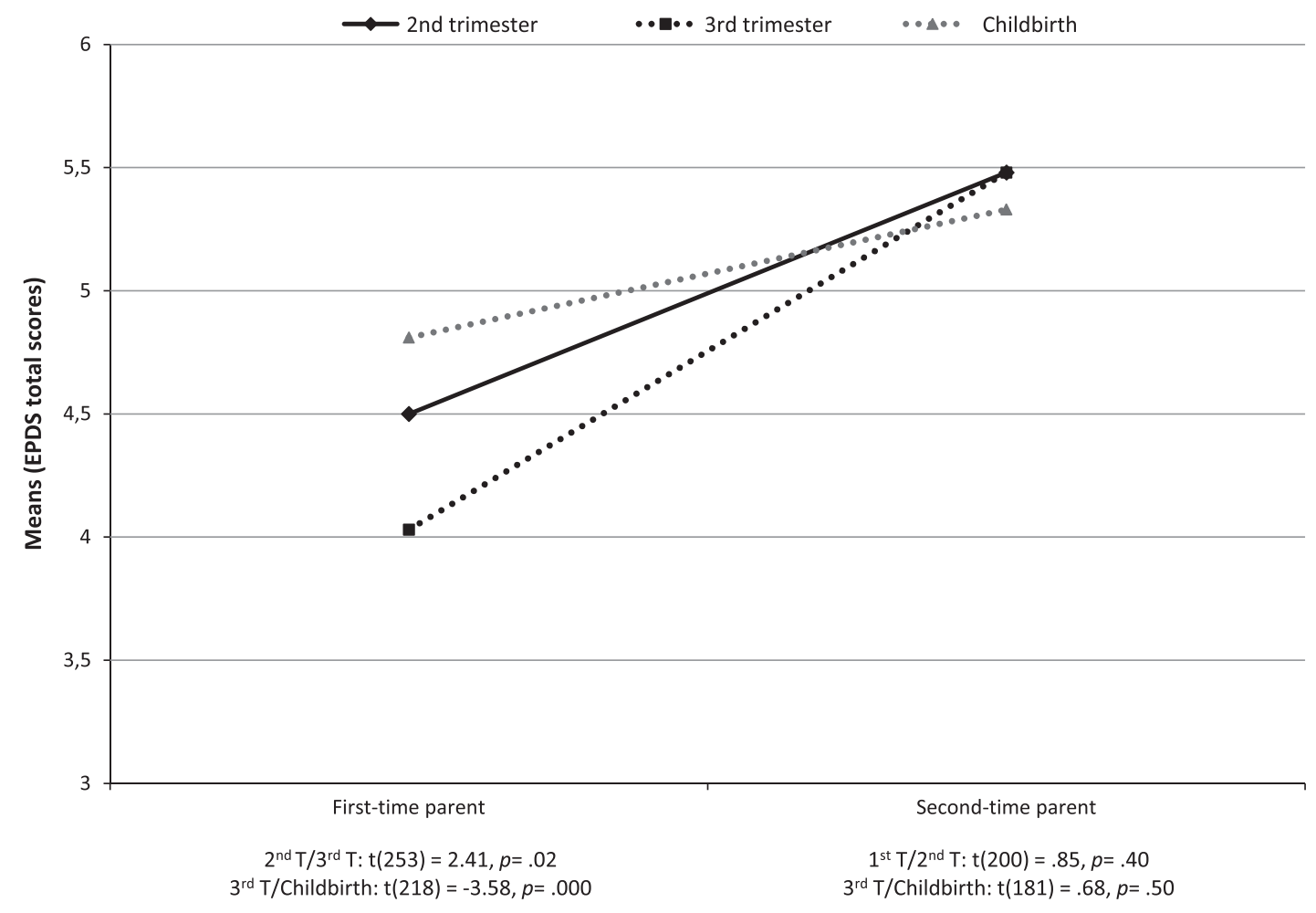

Fig. 4. Interaction between measuring time points and parity for depression symptoms. 
Table 3

Depression from early pregnancy to 3-months postpartum: women and men, 1st and 2nd time parents, and total sample.

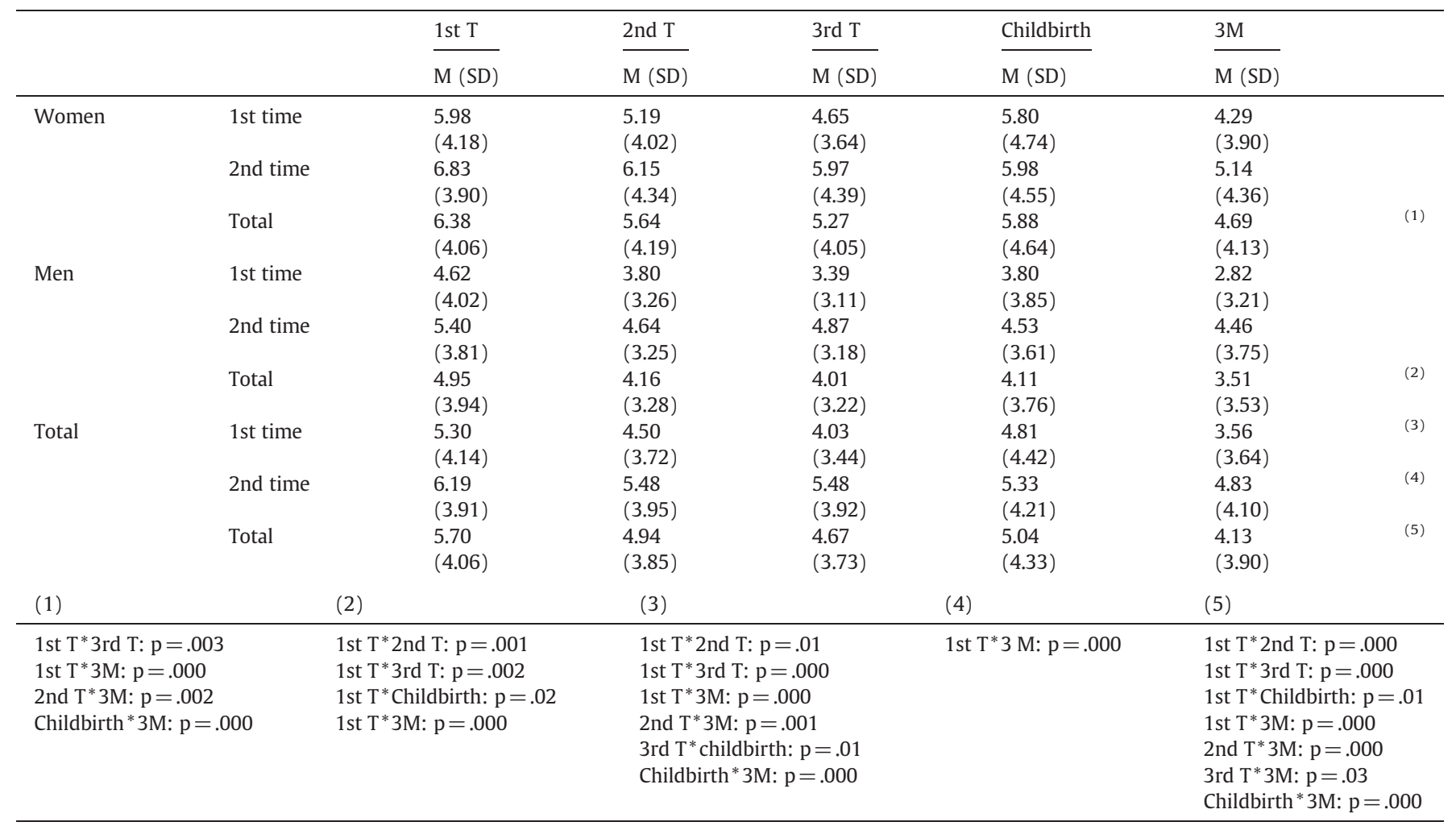

during pregnancy and the postpartum period suggested in the literature, regarding women's anxiety (Boyce et al., 2007; Buist et al., 2003; Ross et al., 2003; Wenzel et al., 2003) and depression (Felice et al., 2004; Ross et al., 2004; Wu et al., 2002), as well as men's anxiety (Quinlivan and Condon, 2005; Huizink et al., 2004) and depression (Ross et al., 2003). But, higher levels of both anxiety and depression symptoms during pregnancy (especially at the 1st trimester) compared to 3-months postpartum were observed in all parents, women or men, as well as first or second-time parents. Our results confirm previous reports that women's (Andersson et al., 2006; Evans et al., 2001; Felice et al., 2004; Heron et al., 2004) and men's (Boyce et al., 2007; Buist et al., 2003; Condon et al., 2004; Perren et al., 2005) anxiety and/or depression is higher before than after childbirth. Showing that mothers and fathers', as well as first and second-time parents' psychological adjustment is enhanced from early pregnancy to 3-months postpartum, as previously suggested: "Although postnatal psychological distress has been widely studied for many years, particularly with a focus on postpartum depression, symptoms of maternal depression, stress and anxiety are not more common or severe after childbirth than during pregnancy" (Kinsella and Monk, 2009, p. 52). In the measure that women and men are more into the transition to parenthood, psychological adjustment is improved and anxiety and depression symptoms diminished. Exemplarily, depression symptoms diminish almost linearly. This can be attributed to the increasing presence of the child in the parents' life, promoting parents' better mood and positive expectations for the future. Additionally, results clearly pointed out that both women and men and first and second-time parents are particularly at-risk during the 1 st pregnancy trimester, suggesting that early gestation developmental tasks (pregnancy acceptation) may be quite difficult for all parents-to-be. As such, similar attention should be given to the parents-to-be psychological adjustment during pregnancy, and particularly during the 1 st pregnancy trimester, as the attention that has been given to the postpartum period.

Significant changes in anxiety and depression symptoms were observed over pregnancy and the first postpartum months, with an increase in anxiety symptoms from the 3rd pregnancy trimester to childbirth and a decrease in depression symptoms from the 1 st to the 2 nd pregnancy trimester. Stability and change in symptoms of depression and anxiety throughout pregnancy and the postpartum period have been referred in the literature (DiPietro et al., 2008; Heron et al., 2004; Skouteris et al., 2009). Despite some parents developing psychological disturbances during this period, for most parents, women or men, first or second-time parents, anxiety and depression symptoms are a sign of a healthy psychological adjustment to the different challenges they face during this transition.

Anxiety and depression had a slightly different pattern of changes. According with what has been pictured out in the literature, in our study anxiety symptoms showed a U pattern during pregnancy, decreasing from the 1st to the 2nd trimester and then increasing from the 2nd trimester with the proximity of childbirth (Lee et al., 2007; Teixeira et al., 2009) followed by a decrease after childbirth (Andersson et al., 2006; Breitkopf et al., 2006; Condon et al., 2004; DiPietro et al., 2006; Heron et al., 2004; Skari et al., 2002). While depression 
diminished almost linearly from pregnancy to 3-months postpartum (Evans et al., 2001; Felice et al., 2004; Heron et al., 2009; Kinsella and Monk, 2009; Lau and Chan, 2009; Limlomwongse and Liabsuetrakul, 2006; Skouteris et al., 2009). The increase in anxiety symptoms from the 3rd pregnancy trimester to childbirth may be related with the proximity of childbirth and the decrease in depression symptoms from the $1 \mathrm{st}$ to the 2nd pregnancy trimester with the emergent presence of the newborn in the parent's life. However, biological adjustments can also be related with these psychological changes.

Women reported more anxiety symptoms than men during pregnancy and at childbirth but not at 3-months postpartum, in agreement with what has been proposed in the literature (Dulude et al., 2002). According to our results, higher anxiety in mothers compared to fathers seems specific of late pregnancy and childbirth. This may be related to the fact that pregnancy/ childbirth particular events are generally perceived as threatening by mothers and might not be as stressful for fathers. On the other hand, the first postpartum months do not appear to be more stressful for women than for men. Both mothers and fathers may have similar difficulties in adapting to challenges associated with taking care of the newborn, reflecting cultural changes associated to the share of parental responsibilities between the mother and the father.

Women reported more depression symptoms than men in all studied moments, as presented in the literature during pregnancy (Dulude et al., 2002; Matthey et al., 2000; Morse et al., 2000; Perren et al., 2005) and the postpartum period (Areias et al., 1996; Ballard and Davis, 1996; Edhborg et al., 2005; Matthey et al., 2000; Morse et al., 2000; Perren et al., 2005). Higher rates of depression are generally found in women compared to men and therefore do not seem to be specific of the pregnancy or postpartum period, contrary to what we have discussed regarding high-anxiety surrounding childbirth. Compared to women, the first pregnancy and postpartum months seems to be as difficult to adjust to for men (Condon et al., 2004; Perren et al., 2005); while compared to men, late pregnancy and childbirth seem to be particularly difficult to adjust to for women. This may be associated with the challenge of specific developmental tasks according to gender: the preparation to childbirth may be more stressful for mothers while the adjustment to parenting and to the new interactions within the family may be as stressful for fathers.

Women and men's patterns of anxiety and depression change across pregnancy and the postpartum period were quite similar, suggesting that developmental tasks and psychological difficulties during the transition to parenthood may not be as different for mothers and fathers. However gender specificities need to be also pointed out. Anxiety in women rises during the 3rd pregnancy trimester and at childbirth and follows a subsequent significant decrease at 3-months postpartum, while a more linear anxiety decrease was observed in fathers from pregnancy to the postpartum period. This suggests that psychological adjustment during pregnancy is particularly difficult compared to the postpartum period adjustment, both for women and men. The 1st pregnancy trimester is equally problematic for men, while for women the 3rd pregnancy trimester and childbirth, as well as the anxiety that the proximity of childbirth may cause, are the most problematic periods. Childbirth also appears as a point of increased symptoms, but mostly only for women and without such impact in men.
Hormonal changes cannot be omitted in the explanation of these results. At the same time, an improvement was observed in the psychological adjustment of both women and men after childbirth. Women and men increased their psychological adjustment from early pregnancy to 3-months postpartum. Suggesting that mothers and fathers are stressed in some similar ways while other difficulties are specific to gender, but they are mainly able to solve and cope positively with the developmental tasks during this transition.

More anxiety and depression symptoms in second-time parents than in first-time parents in all measuring time points, with the exception of anxiety at the 1st trimester and childbirth and depression at childbirth, was pointed out. When anxiety is considered, studies usually report more symptoms in primiparous than in multiparous mothers during pregnancy and the postpartum (Gameiro et al., 2009; Giakoumaki et al., 2009; Hung, 2004). However, our results suggest the contrary in accordance with other studies pointing out higher anxiety in second than in first-time mothers either before (DiPietro et al., 2008) or after childbirth (Skari et al., 2002). When depression is considered, studies usually report either no differences between first and second-time mothers both during pregnancy (Breitkopf et al., 2006; Bunevicius et al., 2009; Dietz et al., 2007; Figueiredo et al., 2007; Lee et al., 2007; Wenzel et al., 2005; Westdahl et al., 2007; Wu et al., 2002) and the postpartum period (Breitkopf et al., 2006; Dietz et al., 2007; Wenzel et al., 2005) or higher depression in primiparous mothers during the postpartum period (Dørheim et al., 2009; Gameiro et al., 2009). Our results show the reverse, with more depression symptoms in second than in first-time mothers, either during pregnancy (DiPietro et al., 2008; Vliegen et al., 2006) and the postpartum period. Some reports have also found higher postpartum depression in mutiparous than in primiparous mothers (HoYen et al., 2007; Righetti-Veltema et al., 1998) and fathers (Condon and Esuvaranathan, 1990).

Data show that pregnancy and the postpartum period get somewhat more difficult the second-time around, at least in this sample of low to medium socio-economic working mothers and fathers. Suggesting the demanding challenges women and men have to face with one more child, namely the reorganization of the previous parental system, the increase of financial responsibilities and the requirements of the professional role. Interestingly our results pointed no differences between first and second-time parents, neither concerning anxiety symptoms at the 1 st trimester, nor concerning anxiety or depression symptoms at childbirth. Additionally, the 1st trimester, and particularly childbirth related developmental tasks or events may be as difficult for second-time as for first-time parents.

A somewhat different pattern of changes was observed in first and second-time parents. In a previous report on this sample, a different pattern between first and second-time parents was found for anxiety but not for depression during pregnancy, with primiparous women and men displaying higher anxiety levels in the 1st than in the 3rd trimester, while in multiparous women and men registered the opposite (Teixeira et al., 2009). It is interesting to note that, despite second-time parents having presented higher anxiety and depression symptoms than first-time parents, a decrease both in anxiety, from the 1 st to the 2 nd trimester, and depression symptoms, from the 2nd to the 3rd trimester, was observed in first-time parents, being not significant for second-time parents, in the 
same way that an increase both in anxiety and depression symptoms was found, from the 3rd trimester to childbirth, in first-time parents, contrary to the decrease obtained both in anxiety and depression symptoms, from the 3rd trimester to childbirth, by second-time parents. Psychological symptoms in first-time parents may be more related to the specific changes associated with pregnancy events than in lasting problems. The proximity of an unknown event, such as childbirth, may be somewhat more stressful for primiparous parents, while the higher psychological difficulties found in second-time parents, over pregnancy and the first postpartum months, can be more related to the need of having to conjugate the challenges of the new child within the pre-existing family system.

Present findings foreground that pregnancy and the postpartum period may be pictured as a period of psychological adjustment/growth, despite the several difficulties that can emerge. Some are specific to women and others specific to men; some are specific to first-time parents and others specific to second-time parents; and some are relating to the transition in all. Other socio-demographics factors seem not to contribute to observed differences at least in this sample. Both empirical evidences need to be addressed. Thus, it is important to refer that the majority of parents exhibit better psychological functioning 3-months after the birth of the child than at the beginning of the pregnancy, and that second-time parents present higher levels of anxiety and depression than first-time parents. According to our results, prevention and intervention programs should better target the first pregnancy trimester and the second-time parents. And more research should be done focusing on the first pregnancy trimester and the second-time parents to better understand difficulties and strengths, similarly to what was done with first-time parents and the postpartum period.

Despite the new important outcomes of this study, some methodological limitations require to be pointed out. We must consider the voluntary nature for the participation in the study, which may have led to a selection bias, in the sense that the women/men who agreed to participate may in fact be those who presented less symptoms of depression or anxiety. This constitutes a particular issue for men, as the rate of fathers-to-be who signed the informed consent was lower when compared to the rate of mothers-to-be. Limitations from using self-report symptom scales rather than clinical interviews and continuous scores rather than cut-off scores need to be considered: the level of possible disorder in the participants is unknown, women and men may have different reporting style, and especially for women some pregnancy specific burden can be confused with the asked symptoms in the questionnaires. In order to better understand the pattern of symptoms over time, namely to relate them with specific developmental challenges or external events, other measures could be added in future studies.

\section{Role of funding source}

Funding for this work was provided by the Operational Program Science and Innovation 2010 (POCI 2010) of the Community Support Board III and supported by the European Community Fund FEDER. (POCI/SAU-ESP/56397/ 2004; Anxiety and depression in women and men during the transition to parenthood: effects on fetal and neo-natal behavior and development).

The sponsors had no further role in the study design; in the collection, analysis and interpretation of data; in the writing of the report; and in the decision to submit the paper for publication.

\section{Conflict of interest}

This work has no conflict of interest.

\section{Acknowledgements}

This work was supported by the Operational Program Science and Innovation 2010 (POCI 2010) of the Community Support Board III and by the European Community Fund FEDER (POCI/SAU-ESP/56397/2004; anxiety and depression in women and men during the transition to parenthood: effects on fetal and neo-natal behavior and development). We gratefully acknowledge the proofreading of this manuscript by the Scientific Editing Program of University of Minho.

\section{References}

Andersson, L., Sundstrom-Poromaa, I., Wulff, M., Astrom, M., Bixo, M., 2006. Depression and anxiety during pregnancy and six months postpartum: a follow-up study. Acta Obstet. Gynecol. Scand. 85, 937-944.

Areias, M.E., Kumar, R., Barros, H., Figueiredo, E., 1996. Comparative incidence of depression in women and men, during pregnancy and after childbirth. Validation of the Edinburgh Postnatal Depression Scale in Portuguese mothers. Br. J. Psychiatry 169, 30-35.

Ballard, C., Davis, R., 1996. Postnatal depression in fathers. Int. Rev. Psychiatry $8(1), 65-71$.

Biaggio, A.M., Natalicio, L., Spielberger, C.D., 1976. The development and validation of an experimental Portuguese form of the State-Trait Anxiety Inventory. In: Spielberger, C.D., Dias-Guerrero, R. (Eds.), Cross-Cultural Research on Anxiety. Hemisphere/Wiley, Washington DC, pp. 29-40.

Boyce, P., Condon, J., Barton, J., Corkindale, C., 2007. First-time fathers' study: psychological distress in expectant fathers during pregnancy. Aust. N. Z. J. Psychiatry 41, 718-725.

Breitkopf, C.R., Primeau, L.A., Levine, R.E., Olson, G.L., Wu, Z.H., Berenson, A.B. 2006. Anxiety symptoms during pregnancy and postpartum. J. Psychosom. Obstet. Gynaecol. 27, 157-162.

Buist, A., Morse, C.A., Durkin, S., 2003. Men's adjustment to fatherhood: implications for obstetric health care. J. Obstet. Gynecol. Neonatal Nurs. $32,172-180$.

Bunevicius, A., Kusminskas, L., Pop, V.J., Pedersen, C.A., Bunevicius, R., 2009. Screening for antenatal depression with the Edinburgh Depression Scale. J. Psychosom. Obstet. Gynaecol. 30 (4), 238-243.

Caliskan, D., Oncu, B., Kose, K., Ocaktan, M.E., Ozdemir, O., 2007. Depression scores and associated factors in pregnant and non-pregnant women: a community-based study in Turkey. J. Psychosom. Obstet. Gynaecol. 28 (4), $195-200$.

Condon, J.T., Esuvaranathan, V., 1990. The influence of parity on the experience of pregnancy: a comparison of first- and second-time expectant couples. Br. J. Med. Psychol. 63 (Pt 4), 369-377.

Condon, J.T., Boyce, P., Corkindale, C.J., 2004. The first-time fathers' study: a prospective study of the mental health and wellbeing of men during the transition to parenthood. Aus.t N. Z. J. Psychiatry 38, 56-64.

Cox, J.L., Holden, J.M., Sagovsky, R., 1987. Detection of postnatal depression. Development of the 10-item Edinburgh Postnatal Depression Scale. Br. J. Psychiatry 150, 782-786.

Deater-Deckard, K., Pickering, K., Dunn, J.F., Golding, J., 1998. Family structure and depressive symptoms in men preceding and following the birth of a child. The Avon Longitudinal Study of Pregnancy and Childhood Study Team. Am. J. Psychiatry 155, 818-823.

Dietz, P.M., Williams, S.B., Callaghan, W.M., Bachman, D.J., Whitlock, E.P., Hornbrook, M.C., 2007. Clinically identified maternal depression before, during, and after pregnancies ending in live births. Am. J. Psychiatry 164, $1515-1520$.

DiPietro, J.A., Novak, M.F., Costigan, K.A., Atella, L.D., Reusing, S.P., 2006 Maternal psychological distress during pregnancy in relation to child development at age two. Child Dev. 77, 573-587.

DiPietro, J.A., Costigan, K.A., Sipsma, H., 2008. Continuity in self-report measures of maternal anxiety, stress, and depressive symptoms from pregnancy through two years postpartum. J. Psychosom. Obstet. Gynaecol. 29, 115-124.

Dørheim, S.K., Bondevik, G.T., Eberhard-Gran, M., Bjorvatn, B., 2009. Sleep and depression in postpartum woman: a population based study. Sleep 32 (7), 847-855.

Dulude, D., Bélanger, C., Wright, J., Sabourin, S., 2002. High-risk pregnancies, psychological distress, and dyadic adjustment. J. Reprod. Infant. Psychol. 20, 101-123. 
Edhborg, M., Matthiesen, A.S., Lundh, W., Widstrom, A.M., 2005. Some early indicators for depressive symptoms and bonding two months postpartum - a study of new mothers and fathers. Arch. Womens Ment. Health $8,221-231$.

Evans, J., Heron, J., Francomb, H., Oke, S., Golding, J., 2001. Cohort study of depressed mood during pregnancy and after childbirth. Br. Med. J. 323, 257-260.

Felice, E., Saliba, J., Grech, V., Cox, J., 2004. Prevalence rates and psychosocial characteristics associated with depression in pregnancy and postpartum in Maltese women. J. Affect. Disord. 82, 297-301.

Field, T., Diego, M., Hernandez-Reif, M., Figueiredo, B., Deeds, O., Contogeorgos, J., Ascencio, A., 2006. Prenatal paternal depression. Infant Behav. Dev. 29 (4), 579-583.

Field, T., Diego, M., Hernandez-Reif, M., Figueiredo, B., Schanberg, S., Kuhn, C., Deeds, O., Contogeorgos, J., Ascencio, A., 2008. Chronic prenatal depression and neonatal outcome. Int. J. Neurosci. 118, 95-103.

Figueiredo, B., Pacheco, A., Costa, R., 2007. Depression during pregnancy and the postpartum period in adolescent and adult Portuguese mothers. Arch. Womens Ment. Health 10, 103-109.

Gameiro, S., Moura-Ramos, M., Canavarro, M.C., 2009. Maternal adjustment to the birth of a child: primiparity versus multiparity. J. Reprod. Infant. Psychol. 27 (3), 269-286.

Giakoumaki, O., Vasilaki, K., Lili, L., Skouroliakou, M., Liosis, G., 2009. The role of maternal anxiety in the early postpartum period: screening for anxiety and depressive symptomatology in Greece. J. Psychosom. Obstet. Gynaecol. 30 (1), 21-28.

Gorman, L.L., O'Hara, M.W., Figueiredo, B., Hayes, S., Jacquemain, F., Kammerer, M.H., Klier, C.M., Rosi, S., Seneviratne, G., Sutter-Dallay, A.L., TCS-PND Group, 2004. Adaptation of the structured clinical interview for DSM-IV disorders for assessing depression in women during pregnancy and postpartum across countries and cultures. Br. J. Psychiatry Suppl. 46, 17-23.

Heron, J., O'Connor, T.G., Evans, J., Golding, J., Glover, V., ALSPAC Study Team, 2004. The course of anxiety and depression through pregnancy and the postpartum in a community sample. J. Affect. Disord. 80, 65-73.

Heron, J., Haque, S., Oyebode, F., Craddock, N., Jones, I., 2009. A longitudinal study of hypomania and depression symptoms in pregnancy and the postpartum period. Bipolar Disord. 11 (4), 410-417.

Ho-Yen, S.D., Bondevik, G.T., Eberhard-Gran, M., Bjorvatn, B., 2007. Factors associated with depressive symptoms among postnatal women in Nepal. Acta Obstet. Gynecol. Scand. 86 (3), 291-297.

Huizink, A.C., Mulder, E.J., Robles de Medina, P.G., Visser, G.H., Buitelaar, J.K., 2004. Is pregnancy anxiety a distinctive syndrome? Early Hum. Dev. 79, 81-91.

Hung, C.H., 2004. Predictors of postpartum women's health status. J. Nurs. Scholarsh. 36 (4), 345-351.

Kinsella, M.T., Monk, C., 2009. Impact of maternal stress, depression and anxiety on fetal neurobehavioral development. Clin. Obstet. Gynecol. 52 (3), 425-440.

Lau, Y., Chan, K.S., 2009. Perinatal depressive symptoms, sociodemographic correlates, and breast-feeding among Chinese women. J. Perinat. Neonatal Nurs. 23 (4), 335-345.

Lee, A.M., Chong, C.S.Y., Chiu, H.W., Lam, S.K., Fong, D.Y.T., 2007. Prevalence, course, and risk factors for antenatal anxiety and depression. Obstet. Gynecol. 110, 1102-1112.

Limlomwongse, N., Liabsuetrakul, T., 2006. Cohort study of depressive moods in Thai women during late pregnancy and 6-8 weeks of postpartum using the Edinburgh Postnatal Depression Scale (EPDS). Arch. Womens Ment. Health 9, 131-138.

Matthey, S., Barnett, B., Ungerer, J., Waters, B., 2000. Paternal and maternal depressed mood during the transition to parenthood. J. Affect. Disord. 60, 75-85.

Morse, C.A., Buist, A., Durkin, S., 2000. First-time parenthood: influences on pre- and postnatal adjustment in fathers and mothers. J. Psychosom. Obstet. Gynaecol. 21 (2), 109-120.

Perren, S., von Wyl, A., Burgin, D., Simoni, H., von Klitzing, K., 2005. Depressive symptoms and psychosocial stress across the transition to parenthood: associations with parental psychopathology and child difficulty. J. Psychosom. Obstet. Gynaecol. 26, 173-183.

Quinlivan, J.A., Condon, J., 2005. Anxiety and depression in fathers in teenage pregnancy. Aust. N. Z. J. Psychiatry 39, 915-920.

Righetti-Veltema, M., Conne-Perréard, E., Bousquet, A., Manzano, J., 1998. Risk factors and predictive signs of postpartum depression. J. Affect. Disord. 49 (3), 167-180.

Ross, L.E., McLean, L.M., 2006. Anxiety disorders during pregnancy and the postpartum period: a systematic review. J. Clin. Psychiatry 67, 1285-1298.

Ross, L.E., Gilbert Evans, S.E., Sellers, E.M., Romach, M.K., 2003. Measurement issues in postpartum depression part 2: assessment of somatic symptoms using the Hamilton Rating Scale for Depression. Arch. Womens Ment. Health 6, 59-64.

Ross, L.E., Sellers, E.M., Gilbert Evans, S.E., Romach, M.K., 2004. Mood changes during pregnancy and the postpartum period: development of a biopsychosocial model. Acta Psychiatr. Scand. 109, 457-466.

Skari, H., Skreden, M., Malt, U.F., Dalholt, M., Ostensen, A.B., Egeland, T., Emblem, R., 2002. Comparative levels of psychological distress, stress symptoms, depression and anxiety after childbirth - a prospective population-based study of mothers and fathers. BJOG 109, 1154-1163.

Skouteris, H., Wertheim, E.H., Rallis, S., Milgrom, J., Paxton, S.J., 2009. Depression and anxiety through pregnancy and the early postpartum: an examination of prospective relationships. J. Affect. Disord. 113 (3), 303-308.

Spielberger, C.D., Gorsuch, R.L., Lushene, R., Vagg, P.R., Jacobs, G.A., 1983. Manual for the State-Trait Anxiety Inventory: STAI (form Y). Selfevaluation questionaire. Palo Alto. Consulting Psychologists Press, Inc.

Teixeira, C., Figueiredo, B., Conde, A., Pacheco, A., Costa, R., 2009. Anxiety and depression during pregnancy in women and men. J. Affect. Disord. 119, 142-148.

Vliegen, N., Luytena, P., Meursa, P., Cluckersa, G., 2006. Adaptive and maladaptive dimensions of relatedness and self-definition: relationship with postpartum depression and anxiety. Pers. Individ. Dif. 41 (3), 395-406.

Wenzel, A., Haugen, E.N., Jackson, L.C., Robinson, K., 2003. Prevalence of generalized anxiety at eight weeks postpartum. Arch. Womens Ment. Health 6, 43-49.

Wenzel, A., Haugen, E.N., Jackson, L.C., Brendle, J.R., 2005. Anxiety symptoms and disorders at eight weeks postpartum. J. Anxiety Disord. 19, 295-311.

Westdahl, C., Milan, S., Magriples, U., Kershaw, T.S., Rising, S.S., Ickovics, J.R., 2007. Social support and social conflict as predictors of prenatal depression. Obstet. Gynecol. 110, 134-140.

Wu, J., Viguera, A., Riley, L., Cohen, L., Ecker, J., 2002. Mood disturbance in pregnancy and the mode of childbirth. Am. J. Obstet. Gynecol. 187, 864-886.

Xie, R., He, G., Koszycki, D., Walker, M., Wen, S.W., 2009. Prenatal social support, postnatal social support, and postpartum depression. Ann. Epidemiol. 19 (9), 637-643. 